\title{
Case Report on Pseudoaneurysm of Brachial Artery: A Rare Complication of Paediatric Supracondylar Humerus Fracture Pinning
}

\author{
Prawesh S Bhandari', Priska Bastola ${ }^{2}$, Dinesh Gurung ${ }^{3}$ \\ 'Department of Orthopaedics, Maharajgunj Medical Campus, Tribhuvan University Teaching Hospital, Institute \\ of Medicine, Maharajgunj, Kathmandu, ${ }^{2}$ Department of Cardiothoracic Vascular Anaesthesiology, Maharajgunj \\ Medical Campus, Manmohan Cardiothoracic Vascular and Transplant Center, Institute of Medicine, Maharajgunj, \\ Kathmandu, ${ }^{3}$ Department of Cardiothoracic and Vascular Surgery, Maharajgunj Medical Campus, Manmohan \\ Cardiothoracic Vascular and Transplant Center, Institute of Medicine, Maharajgunj, Kathmandu
}

\section{Corresponding author:}

\section{Priska Bastola, MBBS, MD}

Department of Cardiothoracic Vascular Anaesthesiology, Manmohan Cardiothoracic Vascular and Transplant Center, Institute if Medicine, Maharajgunj, Kathmandu

Email:priskasb@gmail.com

Submitted : November 7, 2019

Accepted : December 12, 2019

\begin{abstract}
Pseudoaneurysm (PA) of the brachial artery in pediatric supracondylar humerus fracture who have undergone closed reduction and percutaneous pinning is a rare complication. A few cases have been reported in medical literature. This case report sheds some insight into early identification and management outcome of such complication. Rapidly progressive swelling in antecubital fossa in cases of supracondylar fracture who have undergone pinning should arouse suspicion of vascular lesion. It should be timely intervened. Surgical intervention of pseudoaneurysm with reversed saphenous venous graft has shown to have no early vascular complication.
\end{abstract}

Keywords: Case report, supracondylar fracture, pseudoaneurysm, supracondylar fracture

\section{INTRODUCTION}

S upracondylar fracture humerus is a commonly encountered paediatric injury making up $17 \%$ paediatric fractures. ${ }^{1}$ It is the most common elbow fracture in children, accounting for $60 \%$ to $70 \%$ of all pediatric elbow fractures. ${ }^{2}$

Extension type of injury is the most commonly encountered and Gartland type II \& III are managed operatively. When requiring operative treatment, percutaneous smooth pin fixation is the commonest modality applied. ${ }^{3}$

The noted complications of percutaneous pinning have been pin migration beneath the skin necessitating a return to the operating room to remove pins $(1.8 \%)$ and by loss of reduction necessitating a return to the operating room for repinning $(0.6 \%)$. The incidence of ulnar nerve injury due to closed medial pinning is also a feared complication which was seen in $0.3 \%{ }^{4}$

Incidence of pseudoaneurysm of the brachial artery in pediatric population either due to blunt trauma or directly attributed to Kirschner wire has been reported. But these occurrences are rare. This case report will help to add to existing pool of knowledge in identifying and managing such rare condition.

\section{CASE PRESENTATION}

A 13 year old male sustained supracondylar humerus fracture (Gartland type III) following a fall from height (Figure 1A).

The fracture was managed with closed reduction and k-wire fixation on the day of injury and the patient was discharged on the next day with limb immobilized on long arm slab. No signs of vascular or nerve injury was noted either before or after the 

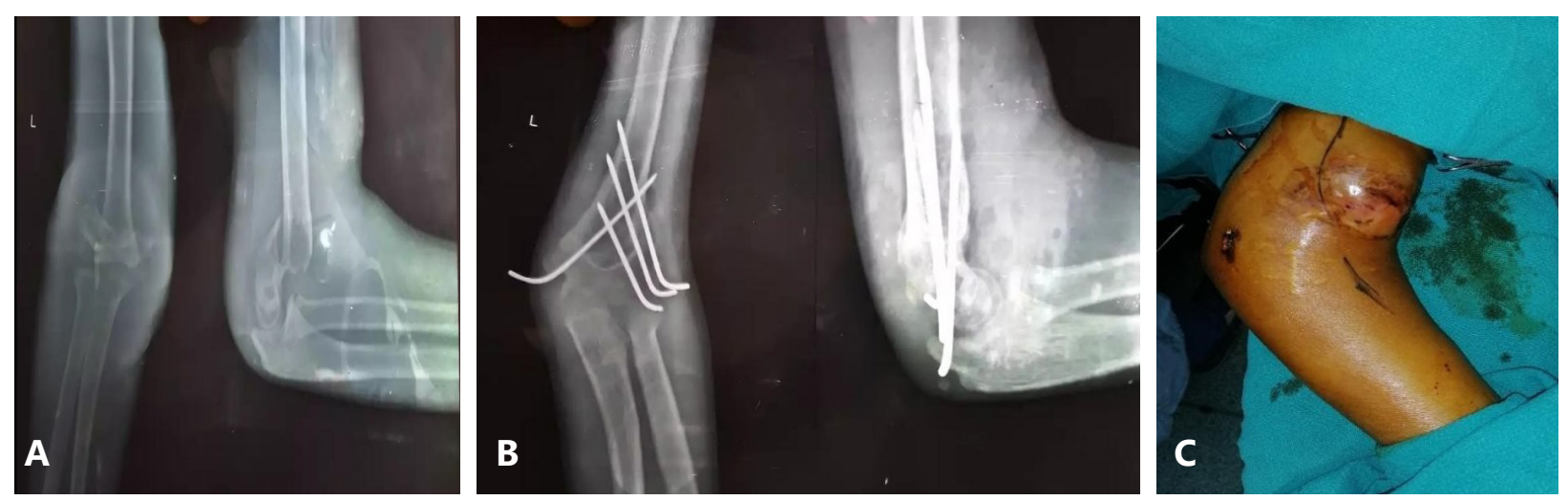

Figure 1A. Anterio Posterior and Lateral radiographic image of Supracondylar humerus Fracture (Gartland type III). Figure 1B. Anterior Posterior and Lateral radiographic image of supracondylar fracture after Kirschner-wire fixation. Figure 1C. Swelling in the cubital fossa

procedure (Figure 1B).

The patient presented 2 weeks post operatively with pain on the cubital region. On clinical evaluation, an expansile swelling of $5 \mathrm{~cm}$ diameter with thrill was found along with a normal radial pulse. A likely diagnosis of brachial artery pseudoaneurysm was made (Figure 1C).

The patient underwent brachial artery repair with interposition reverse saphenous vein graft. After confirming the reduction under image intensifier the k-wires were removed during the vascular repair and the patient was discharged on the third postoperative day.

The patient was followed up eight weeks post vascular repair which showed no features of vascular deficit.

\section{DISCUSSION}

Vascular compromise can be present in up to $20 \%$ of patients with displaced supracondylar humerus fractures. ${ }^{5}$ There have been very few case reports of false aneurysm occurring in supracondylar humerus fracture in children. ${ }^{2,6,7}$

In all of the above cases there is a common mode of presentation which is a persistence of swelling in the cubital fossa after supracondylar fracture which arouses the suspicion of false aneurysm. But the time of presentation is varied ranging from as early as 3 weeks to delayed presentation till 7 months. In our case the patient has reported at the earliest which was second week post injury.

In cases reported by Alberto Hernandez et $\mathrm{al}^{7}$ and Asavamongkolkul $A^{6}$ the cause for PA was the protruding hardware. Got $\mathrm{C}$ et $\mathrm{al}^{2}$ attributed PA to the injury. However author could not clearly attribute the cause of pseudoaneurysm to primary injury caused by the bony spike or iatrogenically created by the penetration of kirshner wire during pinning of the fracture which sometimes requires multiple attempts to place pin properly in order to achieve stability. Luria et al found significant correlation between vascular injury and median nerve deficit in supracondylar humerus fractures. ${ }^{8}$ The absence of median nerve injury at the onset makes it less likely that brachial artery injury might not have been injured in isolation.

There has been an article which has described that there is a discrepancy in the length assessed radiographically and in reality. This study shows that intraoperative fluoroscopic images tend to underestimate actual lengths of smooth pin protruding from bony surfaces by a mean of 1.5 $\mathrm{mm} .{ }^{9}$ Such overestimation if avoided can lessen such complications.

\section{CONCLUSION}

Pinning after closed reduction of pediatric supracondylar fracture is a commonly performed procedure. Pseudoaneurysm can develop both due to penetration by bony spike of fracture or by pin protruding out of anterior cortex. Persistent swelling in the cubital fossa should arouse suspicion of pseudoaneurysm which should be appropriately investigated and managed.

\section{CONFLICT OF INTEREST}

None declared.

\section{REFERENCES}

1. Rockwood CA, Beaty JH, Kasser JR, eds. Rockwood and Wilkins' fractures in children. Wolters Kluwer/Lippincott ; 2010.

2. Got C, Tan TW, Thakur N, Marcaccio Jr EJ, Eberson C, Madom I. Delayed presentation of a brachial artery pseudoaneurysm after a supracondylar humerus fracture in a 6-year-old boy: a case report. Journal of Pediatric Orthopaedics. 2010 


$$
\text { Jan;30(1):57-9 }
$$

3. Ducić $S$, Bumbasirević $M$, Radlović $V$, et al. Displaced supracondylar humeral fractures in children: comparison of three treatment approaches. Srp Arh Celok Lek. 2016;144:46-51.

4. Bashyal RK, Chu JY, Schoenecker PL, Dobbs MB, Luhmann SJ, Gordon JE. Complications after pinning of supracondylar distal humerus fractures. Journal of Pediatric Orthopaedics. 2009 Oct;29(7):704-8.

5. Harris LR, Arkader A, Broom A, Flynn J, Yellin J, Whitlock P, Miller A, Sawyer J, Roaten J, Skaggs DL, Choi PD. Pulseless Supracondylar Humerus Fracture With Anterior Interosseous Nerve or Median Nerve Injury-An Absolute Indication for Open Reduction?. Journal of Pediatric Orthopaedics. 2019 Jan;39(1):e1-7.5

6. Asavamongkolkul A, Ruangsetakit C. False aneurysm of the brachial artery in supracondylar fracture treated with Kirschner wire fixation: a case report. Injury. 2001 Apr 1;32(3):256-7.

7. Hernandez-Fernandez A, Vélez $R$, Soldado $F$, Fontecha CG, Barber I, Aguirre M. false Aneurysm of the Brachial Artery Secondary to K-wire Fixation of a Supracondylar Fracture of the Humerus. European Journal of Pediatric Surgery. 2013 Jun;23 (03):251-3.

8. Luria S, Sucar A, Eylon S, et al. Vascular complications of supracondylar humeral fractures in children. J Paediatr Orthop B. 2007;16:133e43

9. Kenney S, Schlechter J. Do fluoroscopic and radiographic images underestimate pin protrusion in paediatric supracondylar humerus and distal radius fractures? A synthetic bone model analysis. Journal of children's orthopaedics. 2019 Feb $1 ; 13(1): 57-61$. 